\title{
CFD PREDICTION OF GAS-LIQUID FLOW IN AN AERATED STIRRED VESSEL USING THE POPULATION BALANCE MODEL
}

\author{
Zbyněk Kálal $^{1}$, Milan Jahoda ${ }^{1}$, Ivan Fořt ${ }^{2}$ \\ ${ }^{1}$ Institute of Chemical Technology, Prague, Technická 5, 16628 Prague 6, Czech Republic \\ ${ }^{2}$ Czech Technical University in Prague, Technická 4, 16607 Prague 6, Czech Republic
}

\begin{abstract}
The main topic of this study is the experimental measurement and mathematical modelling of global gas hold-up and bubble size distribution in an aerated stirred vessel using the population balance method. The air-water system consisted of a mixing tank of diameter $T=0.29 \mathrm{~m}$, which was equipped with a six-bladed Rushton turbine. Calculations were performed with CFD software CFX 14.5. Turbulent quantities were predicted using the standard $k-\varepsilon$ turbulence model. Coalescence and breakup of bubbles were modelled using the homogeneous MUSIG method with 24 bubble size groups. To achieve a better prediction of the turbulent quantities, simulations were performed with much finer meshes than those that have been adopted so far for bubble size distribution modelling. Several different drag coefficient correlations were implemented in the solver, and their influence on the results was studied. Turbulent drag correction to reduce the bubble slip velocity proved to be essential to achieve agreement of the simulated gas distribution with experiments. To model the disintegration of bubbles, the widely adopted breakup model by Luo \& Svendsen was used. However, its applicability was questioned.
\end{abstract}

Keywords: CFD, mixing tank, gas-liquid, drag correction, bubble size distribution

\section{INTRODUCTION}

Fluid flow of one-phase systems in mechanically agitated tanks is presently well explored both experimentally and using simulations. A detailed description of the dynamic behaviour and the determination of hydrodynamical variables of multiphase systems is a more difficult task because of the complexity of the interactions between phases. The interactions include various mechanisms of momentum exchange, such as drag, lift, virtual mass, Basset and turbulent dispersion forces. However, in gas-liquid stirred tanks, the drag force has been shown to be the most significant contributor, while the others can be neglected during calculations (Scargiali et al., 2007). The basic correlation for particle drag determination is the Schiller-Naumann model. In fact, this model was derived for movement of a single solid, spherical particle in stagnant fluids, and is therefore valid only for small, separate bubbles moving in a non-turbulent fluid. These bubbles can be considered to be perfectly spherical. Thus, modified models that take into account significant deformations of larger bubbles and a decrease of drag in dense dispersions (Ishii and Zuber, 1979; Tomiyama et al., 1998) or interactions of bubbles with turbulent eddies (Bakker and van den Akker, 1994; Brucato et al., 1998; Montante et al., 2007) have been derived.

A constant bubble size is often considered in calculations to decrease computational requirements because engagement of the bubble size distribution (BSD) modelling significantly increases the 
computation time. However, modelling of the BSD is essential for the correct prediction of process characteristics of a system. It strongly influences gas hold-up or mass transfer between phases, which is a function of the interfacial area and is thus also a function of bubble size. A lot of published works that include the BSD modelling have focused on the investigation of bubble columns up to now. However, the breakup and coalescence models validated at such mild and homogeneous turbulence conditions may not be adequate for use in much more turbulent and inhomogeneous systems such as stirred tanks.

To determine the BSD, the population balance equation (PBE) must be solved. It describes the bubble population evolution and can be solved via various approaches. The most widely explored approaches include the Class Methods (CM) and the Methods of Moments (MOM). Typical examples of the former group are the fixed pivot technique (Kumar and Ramkrishna, 1996) and the Multiple Size Group (MUSIG) method (Lo, 1996), the latter group is represented especially by the Quadrature Method of Moments (QMOM) and its variations (Marchisio and Fox, 2013). Selma et al. (2010) confronted the $\mathrm{CM}$ and modified QMOM with experimental results measured both in a bubble column and in a stirred tank. The CM was used with 15 classes as a good trade-off between accuracy and computational time. Both the CM and the QMOM were able to deal with the PBE and to provide reliable results. The essential factor is rather the choice of the appropriate coalescence and breakup models. While the coalescence models are usually derived on the basis of the kinetic theory of gases and thus exhibit similar behaviour, various breakup models differ significantly in the predicted breakup frequency or in the shape of the daughter size distribution (DSD) function. The most frequently used breakup model was given by Luo and Svendsen (1996). It has been applied by many authors for modelling of the BSD in stirred tanks (e.g., Ahmed et al., 2010; Kerdouss et al., 2008; Montante et al., 2008; Ranganathan and Sivaraman, 2011; Selma et al., 2010). Therefore, we focus on reviewing this breakup model in our work. A comparison with different breakup models will be presented in our upcoming study.

The breakup and coalescence kernels are strong functions of the turbulent dissipation energy. A correct prediction of turbulent quantities is thus essential for BSD modelling. It has been stated many times that the $k-\varepsilon$ turbulence model underestimates the turbulent quantities, which results in underestimation of the power number computed from the integral of the turbulent dissipation rate. Coroneo et al. (2011) tested four different computational grids ranging from 270,000 to 6.6 million cells on a single-phase system and concluded that the turbulent quantities prediction may be substantially improved by reducing the spatial discretisation size. In recent years, the traditional approach was to use computational meshes with less than half a million cells for the BSD modelling in stirred tanks. However, much finer grids than those that have been usually adopted so far for RANS simulations are obviously required for accurate prediction of the turbulent quantities. Coroneo et al. (2011) also found that the influence of the discretisation scheme is critical. They compared the first order upwind, second order upwind and QUICK scheme. The first order discretisation scheme proved to be much less accurate than the other two and it contributed to the underestimation of the turbulent quantities. The other two discretisation schemes provided the same results. On the other hand, grid refinement and choice of the discretisation scheme do not have a significant effect on velocity field and flow number predictions. These results indicate that coarser grids may be used if only the mean fluid flow is in focus but that very fine grids are required for accurate prediction of the turbulent quantities (Deglon and Meyer, 2006). Aubin et al. (2004) studied the difference between the flow fields calculated with the stationary and the time dependent modelling approaches and found it to be negligible.

The objective of this article is to present experimental measurements and CFD simulations of gas holdup and bubble size distribution in a stirred tank equipped with a Rushton turbine and containing an aerated water charge. Attention is also focused on the accuracy of turbulent field prediction depending on the computational mesh size. The paper gives a summary and comparison of various drag coefficient correlations given in the literature and a thorough review of the generally accepted breakup model by Luo. 


\section{EXPERIMENTAL SETUP}

Experiments were performed in a cylindrical vessel with an inner diameter $T=0.29 \mathrm{~m}$, which was filled with tap water to height $T$ (Fig. 1). Four baffles with widths of $T / 10$ and thickness of $10 \mathrm{~mm}$ were placed evenly around the perimeter of the vessel wall. The tank was equipped with a standard sixbladed Rushton turbine of diameter $T / 3$, the distance of which from the vessel bottom was $T / 3$. The turbine was mounted on a central shaft that rotated with constant speed $300 \mathrm{rpm}$. A ring sparger was positioned halfway between the vessel bottom and the lower edge of the impeller. The upper side of the ring sparger contained six point outlets with diameters of $1.5 \mathrm{~mm}$ through which air was introduced into the system. The volumetric gas flow rate was varied from $41 / \mathrm{min}$ to $81 / \mathrm{min}(0.2$ and $0.4 \mathrm{vvm})$.

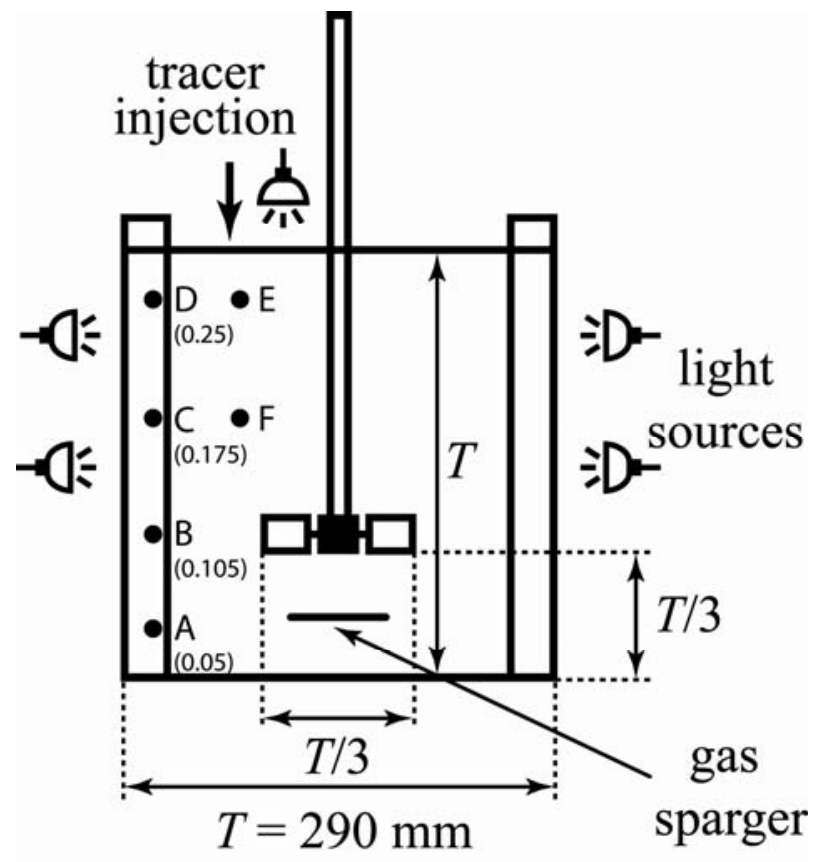

Fig. 1. Geometry of the stirred tank with six points for the BSD measurement (A-F); the numbers in parentheses refer to vertical distance from the vessel bottom $[\mathrm{m}]$

Because the measurement of local gas hold-up is complicated and requires sophisticated equipment, such as CT (Computed Tomography) (Khopkar et al., 2005), only global gas hold-up was measured based on the principle of liquid height elevation. The liquid surface in the tank fluctuates during stirring and so does not allow a simple reading of the elevation. Therefore, a sequence of photographs of the liquid surface at the vessel wall was acquired under ungassed and gassed conditions. Based on the image analysis, the average position of the surface was determined for each set of conditions, and gas hold-up was calculated according to the difference in the water levels.

The determination of the BSD was accomplished using photographic analysis. The main benefit of this method compared with other methods is that bubbles of practically all sizes can be detected from highresolution photographs. On the other hand, the photographing and subsequent evaluation of dense dispersions is generally difficult due to overlapping of bubbles (Laakkonen et al., 2007). Nevertheless, this method appears to be the most convenient and easy method to use.

The mixing tank was placed in a rectangular, glass vessel filled with water, which prevented distortion of the view through a rounded wall. A set of lights was positioned on both sides of the tank and above the free liquid surface. These lights illuminated only the plane where bubble snapshots were taken so that the unwanted background was significantly suppressed, which facilitated the identification of bubbles situated in the plane. The BSD was investigated at six areas (see Fig.1), which were all centred at the vertical plane between baffles. The dimensions of the areas were $2 \times 2 \times 1 \mathrm{~cm}$ 
(width $\times$ height $\times$ depth). Positions A-D were located in the vicinity of the wall (at a distance of $2 \mathrm{~cm}$ ); points $\mathrm{E}$ and $\mathrm{F}$ were halfway between the vessel wall and the impeller shaft. During the taking of photographs, a needle of known dimensions was placed in a desired position; the needle simultaneously served both as the point at which the image was focused and as a scale for evaluating the photographs. Because the fluid moves in the mixed tank at a considerable speed, it was necessary to use a short exposure time, $1 / 1250 \mathrm{~s}$, to gain sharp contours of the bubbles. Furthermore, the depth of field was adjusted to obtain sharp outlines of only the bubbles that were located within the plane of interest. Bubbles that were outside of this particular plane were blurred and therefore not considered in the analysis. Captured bubbles were manually identified, and their size was evaluated via image analysis using the NIS-Elements software. The experimental error was $0.05 \mathrm{~mm}$.

The equivalent diameter of a spherical bubble was determined from the area of the circle, which was set equal to the area of the bubble evaluated using image analysis. We note that some authors have considered bubbles as prolate (Rakoczy and Masiuk, 2009) or oblate (Laakkonen et al., 2007) ellipsoids. They computed the equivalent diameter of a spherical bubble from the volume of a sphere, which was equal to the volume of the ellipsoids. However, because the third dimension of a bubble, and therefore the direction in which a bubble is prolonged (i.e., whether it is prolate or oblate), is impossible to determine from planar photographs, the procedure used in this study appears to be a good trade-off because it provides a larger equivalent diameter than that obtained under the assumption of a prolate ellipsoid and a smaller equivalent diameter than that obtained under the assumption of an oblate ellipsoid.

The determination of the size of bubbles at the gas inlet was also required for the simulation because this parameter represents one of the boundary conditions of the numerical model. However, even at low gas flow rates, gas jetting occurred, which made it impossible to identify the bubble size. Therefore, the flow rate was reduced to a value at which separated bubbles were formed at the outlet of the gas sparger. Their size was used as input parameter of the model.

\section{GOVERNING EQUATIONS}

\subsection{Flow equations}

In the Eulerian model, all phases are considered to be interpenetrating continua. The phase volume fractions, velocity components and pressure are computed from the continuity equations, the NavierStokes equations and the phase volume fraction summation over all phases. The most significant interphase force in stirred tanks is the drag force, $\vec{F}_{D, i j}$, that results from the movement of a bubble through a fluid:

$$
\vec{F}_{D, g l}=\frac{3 \alpha_{l} \alpha_{g} \rho_{l} c_{D}}{4 d_{b}}\left|\vec{U}_{g}-\vec{U}_{l}\right|\left(\vec{U}_{g}-\vec{U}_{l}\right)
$$

The choice of the drag coefficient, $c_{D}$, correlation can significantly affect the results obtained using CFD simulations. A common model that defines the drag coefficient is the model by Schiller and Naumann (1935):

$$
c_{D}=\max \left(\frac{24}{\operatorname{Re}}\left(1+0.15 \operatorname{Re}^{0.687}\right), 0.44\right)
$$

However, this model was derived for separate, rigid, spherical particles; it is therefore valid only for small bubbles in dilute dispersions. At larger particle Reynolds numbers, bubbles are distorted and 
become ellipsoidal or cap-shaped. In the distorted regime, the drag coefficient is dependent on the particle shape, which can be expressed through the Eötvös number. Correlations that take into account the bubble shape have been given by Ishii and Zuber (1979) and by Tomiyama et al. (1998). These correlations are valid for contaminated systems, where bubbles behave as objects with immobile surfaces, see the following equations:

$$
\begin{gathered}
c_{D}=\max \left(\frac{24}{\operatorname{Re}}\left(1+0.15 \mathrm{Re}^{0.687}\right), \min \left(\frac{2}{3} E o^{1 / 2}, \frac{8}{3}\right)\right) \\
c_{D}=\max \left(\frac{24}{\operatorname{Re}}\left(1+0.15 \mathrm{Re}^{0.687}\right), \frac{8}{3} \frac{E o}{E o+4}\right)
\end{gathered}
$$

The main drawback of all the previously mentioned drag coefficient correlations is that they are valid only in stagnant fluids. In turbulent flows, which are typical for stirred tanks, the bubble rise velocities are significantly reduced in comparison with rise velocities in stagnant fluids, which introduces the need for turbulent drag correction. Brucato et al. (1998) accounted for an increase in the solid particle drag due to turbulence by adding an extra term to a drag coefficient in stagnant fluids, $c_{D, 0}$ :

$$
c_{D}=c_{D, 0}+C_{1} c_{D, 0}\left(\frac{d_{b}}{\eta}\right)^{3}
$$

Khopkar et al. (2005) modified this correlation for use in gas-liquid systems by adjusting the model constant $C_{1}=6.5 \cdot 10^{-6}$.

Bakker and van den Akker (1994) based their model on the introduction of the turbulent viscosity in the computation of a modified Reynolds number, and they validated it for gas-liquid systems. The modified criterion enters the calculation of an arbitrary drag coefficient correlation that was originally derived for movement in stagnant fluids:

$$
\operatorname{Re}_{\bmod }=\frac{\rho_{l}\left|\vec{U}_{g}-\vec{U}_{l}\right| d_{b}}{\mu_{l}+C_{2} \mu_{t, l}}
$$

where $C_{2}$ is an adjustable constant.

In systems characterised only by gravitational body forces and zero fluid velocity, the particle drag coefficient can be simply expressed from the force balance applied to the particle:

$$
c_{D}=\frac{4 g d_{b}\left(\rho_{l}-\rho_{g}\right)}{3 \rho_{l} U_{t}^{2}}
$$

where $U_{t}$ is the terminal velocity of bubbles. An isolated air bubble with a diameter less than $5 \mathrm{~mm}$ moving in still water presents a terminal velocity of approximately $25 \mathrm{~cm} / \mathrm{s}$. Montante et al. (2007) proposed to decrease this value to capture the decrease of the bubble slip velocity induced by turbulence effects. Although the value is dependent on the local turbulent intensity, a simplified approach that assumes a constant terminal velocity of $12 \mathrm{~cm} / \mathrm{s}$ was applied by the authors.

\subsection{Turbulence equations}

For turbulence modelling, the $k-\varepsilon$ turbulence model is applied to the continuous phase. This twoequation model assumes turbulence isotropy and consists of equations for the turbulent kinetic energy, 
$k$, and the turbulent dissipation energy, $\varepsilon$ (ANSYS, Inc., 2012). The turbulent quantities are used for calculation of the shear turbulent viscosity of the liquid:

$$
\mu_{t s, l}=C_{\mu} \rho_{l} \frac{k^{2}}{\varepsilon}
$$

In bubbly flows, the bubble-induced turbulence may significantly affect the total turbulent viscosity of the continuous phase, $\mu_{t, l}$. Sato and Sekoguchi (1975) proposed an expression for the bubble-induced turbulent viscosity, $\mu_{t b, l}$, in the form:

$$
\mu_{t b, l}=0.6 \rho_{l} \alpha_{g} d_{b}\left|\vec{U}_{g}-\vec{U}_{l}\right|
$$

The effective viscosity is then the sum of all viscosity contributions:

$$
\mu_{e f f, l}=\mu_{l}+\mu_{t s, l}+\mu_{t b, l}
$$

No equations for turbulence in the dispersed phase are introduced. The calculation of the gas turbulent viscosity is based on a simple assumption of the turbulent kinematic viscosity equality of both phases, which gives

$$
\mu_{t, g}=\frac{\rho_{g}}{\rho_{l}} \mu_{t, l}
$$

The gas effective viscosity is then again the sum of the laminar and turbulent viscosities.

\subsection{Population balance model}

To capture changes in the particle size, an additional equation is necessary to include in the model. This equation is referred to as the population balance equation. It is a complicated integro-partial differential equation. Therefore, various numerical methods are used to deal with this problem.

The MUSIG method is based on the discretisation of the continuous size range of a particle population to a finite number of size intervals (classes, groups). Every interval is represented by a specific value for the particle size $V_{i}$, and each particle can interact with particles in other groups. Due to the coalescence and breakup phenomena, particles with volumes $V$ that lie somewhere between two neighbouring nodes $V_{i}$ and $V_{i+1}$ can also be produced. This problem is solved by splitting the particles into two neighbouring groups. A transport equation for the volumetric fraction of class $i$ in gas, $f_{i}$, must be solved for each interval and it is obtained by integrating the PBE over a size interval:

$$
\frac{\partial}{\partial t}\left[\alpha_{g} \rho_{g} f_{i}\right]+\nabla \cdot\left[\alpha_{g} \rho_{g} \vec{U}_{g} f_{i}\right]=B_{a g, i}-D_{a g, i}+B_{b r, i}-D_{b r, i}
$$

where $B_{a g, i}, B_{b r, i}$ express the birth rates of bubbles of size $V$ to $V+\mathrm{d} V$ due to coalescence and breakup, and $\mathrm{D}_{a g, i}, D_{b r, i}$ are the corresponding particle death rates. To describe these source terms, models that describe the coalescence rate between bubbles of size $V_{i}$ and $V_{j}, a\left(V_{i}, V_{j}\right)$, the breakup rate of bubbles of size $V_{i}, g\left(V_{i}\right)$, and the DSD function of mother particles of size $V_{i}$ breaking into particles of size $V_{j}$, $\beta\left(V_{i} \mid V_{j}\right)$, must be introduced. For the sake of brevity, the mathematical description of the source terms is not presented here and we refer to the corresponding literature (ANSYS, Inc., 2012; Lo, 1996).

Coupling of the population balance model (PBM) with hydrodynamics is done via the Sauter mean diameter (SMD), $d_{32}$, which is obtained from computed values of $f_{i}$ : 


$$
d_{32}=\frac{\sum_{i} f_{i}}{\sum_{i}\left(f_{i} / d_{i}\right)}
$$

The flow field, phase fractions and turbulent quantities are calculated by CFD and they are used to solve the PBM. The resulting bubble size distribution is used to calculate the SMD in each computational cell. This value of the SMD then enters the calculation of the drag coefficient and thus the interphase forces (Eq.(1)). This is the CFD-PBM coupling.

This approach results in the fact that in the homogeneous MUSIG model bubbles of all sizes are characterised by one diameter value in each computational cell and thus share the same velocity. A more powerful but even more computationally demanding method for solving the PBE is the inhomogeneous MUSIG. This method is based on the division of bubbles into two or more velocity groups, which behave like separate phases with their own velocity fields. These are then further subdivided into size groups (Frank et al., 2008).

\section{Bubble coalescence}

The coalescence rate is directly proportional to the collision frequency of bubbles, $\omega_{a g}$, and the collision efficiency, $P_{a g}$ :

$$
a\left(V_{i}, V_{j}\right)=\omega_{a g}\left(V_{i}, V_{j}\right) P_{a g}\left(V_{i}, V_{j}\right)
$$

Various mechanisms of coalescence are presently known (Liao and Lucas, 2010). Most of these mechanisms are usually neglected, especially in stirred tanks with high levels of turbulence, when compared with turbulence-induced coalescence.

One of the most widely used coalescence models is that given by Prince and Blanch (1990):

$$
\begin{gathered}
\omega_{a g}\left(V_{i}, V_{j}\right)=F_{a g} \frac{\pi}{4} \sqrt{2}\left(d_{i}+d_{j}\right)^{2} \varepsilon^{1 / 3}\left(d_{i}^{2 / 3}+d_{j}^{2 / 3}\right)^{1 / 2} \\
P_{a g}\left(V_{i}, V_{j}\right)=\exp \left(-\frac{\rho_{l}^{1 / 2} r_{i j}^{5 / 6} \varepsilon^{1 / 3}}{4 \sigma^{1 / 2}} \ln \frac{h_{0}}{h_{f}}\right)
\end{gathered}
$$

where

$$
r_{i j}=\left(\frac{1}{2}\left(\frac{1}{r_{i}}+\frac{1}{r_{j}}\right)\right)^{-1}
$$

$F_{a g}$ is the calibration factor, $\sigma$ is the surface tension, $h_{0}$ is the initial thickness of a film that separates the coalescing bubbles, and $h_{f}$ is the critical thickness at which rupture of the film and thus coalescence occurs $\left(10^{-4}\right.$ and $10^{-8} \mathrm{~m}$, respectively).

\section{Bubble breakup}

The breakup model developed by Luo and Svendsen (1996) is based on the assumption of isotropic turbulence and considers only binary breakup. The authors assumed that to induce breakup, the energy of the incoming eddy must be larger than the increase in the surface energy due to bubble breakup. The significant difference between this model and its predecessors is that it provides not only the overall breakup rate but also the partial breakup rate, i.e., the breakup rate for a mother particle of size $V_{i}$ splitting into a particle of size $V_{j}$ and its complement. This partial breakup rate is expressed as 


$$
g\left(V_{i}, V_{j}\right)=F_{b r} 0.9238 \frac{\varepsilon^{1 / 3}}{d_{i}^{2 / 3}}\left(1-\alpha_{g}\right) \int_{\xi_{\min }}^{1} \frac{(1+\xi)^{2}}{\xi^{11 / 3}} \exp \left(-\frac{12 c_{f} \sigma}{\beta \rho_{l} \varepsilon^{2 / 3} d_{i}^{5 / 3} \xi^{11 / 3}}\right) d \xi
$$

where $\xi$ is the dimensionless eddy size, $c_{f}$ is the coefficient of change in the surface area, $\beta$ is a constant equal to 2 , and $F_{b r}$ is a calibration coefficient:

$$
c_{f}=f^{2 / 3}+(1-f)^{2 / 3}-1, \quad f=\frac{V_{j}}{V_{i}}, \quad \xi_{\min }=11.4 \frac{\eta}{d_{i}}, \quad \eta=\left[\frac{\left(\frac{\mu_{l}}{\rho_{l}}\right)^{3}}{\varepsilon}\right]^{1 / 4}
$$

where $\eta$ is the Kolmogorov microscale.

The integral in Eq.(18) can be approximated using the incomplete gamma function. We can find seemingly different forms of the approximation in the literature. This is because authors have used different definitions of the incomplete gamma function. Alopaeus et al. (1999) gave the approximation (Eq.(20) in their work), where the incomplete gamma function was defined as:

$$
\Gamma(a, z)=\int_{z}^{\infty} e^{-t} t^{a-1} d t
$$

The total breakup frequency of particles of size $V_{i}$ is then computed as an integral over all possible daughter particle sizes:

$$
g\left(V_{i}\right)=\frac{1}{2} \int_{0}^{1} g\left(V_{i,} V_{j}\right) d f
$$

A necessary part of each breakup model is the DSD function that describes the probability of formation of a daughter particle of size $V_{j}$ by breakup of a mother particle of size $V_{i}$. A great advantage of the model by Luo is that the DSD function is derived directly from the model for the breakup rate and is found by normalising the partial breakup rate by the overall breakup rate:

$$
\beta\left(V_{i} \mid V_{j}\right)=\frac{1}{V_{i}} \frac{g\left(V_{i}, V_{j}\right)}{g\left(V_{i}\right)}=\frac{2 \int_{\xi_{\min }}^{1} \frac{(1+\xi)^{2}}{\xi^{11 / 3}} \exp \left(-\frac{12 c_{f} \sigma}{\beta \rho_{l} \varepsilon^{2 / 3} d_{i}^{5 / 3} \xi^{11 / 3}}\right) d \xi}{V_{i} \int_{0}^{1} \int_{\xi_{\min }}^{1} \frac{(1+\xi)^{2}}{\xi^{11 / 3}} \exp \left(-\frac{12 c_{f} \sigma}{\beta \rho_{l} \varepsilon^{2 / 3} d_{i}^{5 / 3} \xi^{1 / 3}}\right) d \xi d f}
$$

\section{COMPUTATIONAL DOMAIN AND NUMERICAL TECHNIQUES}

The geometry of the studied system was created in the preprocessor DesignModeler. The domain was classically divided into a rotating part that contained the impeller, and a stationary part, where walls, baffles and the gas sparger were situated. For mesh generation, another preprocessor was used: Meshing. Structured computational grids of four different sizes were tested - a basic mesh with 185,000 cells (Grid 1), a refined mesh with 570,000 cells (Grid 2), and two fine meshes that contained 2 million (Grid 3) and 3.5 million cells (Grid 4).

CFD simulations were performed using the software CFX 14.5. For numerical modelling of multiphase flow, the most complex Eulerian model was chosen so that a set of transport equations was solved for 
each phase. Because the system was in fully turbulent regime $\left(\operatorname{Re}=5.06 \times 10^{4}\right)$, the standard $k-\varepsilon$ turbulence model was used. The impeller motion was modelled using the MRF (Multiple Reference Frames) method. The walls were treated as no-slip boundaries and the near-wall flow was resolved using the scalable wall functions (ANSYS, Inc., 2012). The upper part of the domain was set as a degassing boundary, which is seen by the continuous phase as a free-slip wall and as an outlet by the dispersed phase. The system was considered as isothermal, and, because of the negligible solubility of air components, transport between phases was omitted.

According to the experimental measurements of bubbles leaving the sparger, the bubble diameter at the inlet was set to $8 \mathrm{~mm}$. The PBE was solved using the MUSIG method with 24 bubble size groups in the range from 0.1 to $16 \mathrm{~mm}$, which required twenty-four additional equations to be solved. The maximum size of this range was chosen with regard to the fact that in the high gas hold-up region above the sparger intensive coalescence occurs and so large bubbles are formed. The drag coefficient correlations were implemented in the solver through user-defined functions. The turbulent dispersion force was included in the model, other interaction forces were neglected.

The second order upwind discretisation scheme was adopted for the convective terms of the governing equations. A substantial under-relaxing was applied in steady-state simulations. This ensured, in combination with the fully implicit solver, smooth convergence to steady-state. At the end of every simulation the residuals of all variables were well below $10^{-4}$. Besides, the global gas hold-up in the system was monitored and the pseudo-steady-state was assumed to be achieved when this quantity remained constant.

\section{RESULTS AND DISCUSSION}

\subsection{Turbulent dissipation energy and power numbers}

The accuracy of the prediction of the turbulent dissipation energy was judged by the computed power number. The power number of the standard six-bladed Rushton turbine in one-phase systems was determined from the correlation given by Rutherford et al. (1996). The simulated power numbers were calculated from the torque on the impeller and on the baffles and from the integral of the turbulent dissipation rate over the whole domain. The power numbers computed via the three different approaches are summarised and compared with the value obtained from the correlation in Fig. 2. The results confirm that the turbulent dissipation energy prediction improves with the increasing number of computational cells. The finest grid underestimates the power number computed from the overall dissipation energy by approximately $18 \%$ while the error of the coarsest grid is nearly $60 \%$. On the other hand, the power numbers calculated via the other two approaches are in good agreement with the correlation even for Grid 2.

Cui et al. (1996) collected gassed power consumption data of several authors and evaluated them all together. The resulting correlation was used for estimation of the power number for gassed system. The predicted decrease in the power number was about $11 \%$ for the lower gas flow rate and $22 \%$ for the larger gas flow rate. Similarly as in the one-phase system, the differences in the power numbers computed via different approaches decrease as the grid becomes denser (Fig. 3). The plot for the higher gas flow rate is qualitatively identical; for the sake of brevity it is not presented. On the basis of these results we can conclude that the underestimation of the power number computed from the overall dissipation energy may be caused by numerical errors rather than by the turbulence model itself. Further refinement of the computational grid may bring further improvement in the prediction of the turbulent quantities but the computational cost would become unbearable. Because the improvement in 
the turbulent field prediction for Grid 4 was only minor in comparison with Grid 3, at the cost of doubling the computational time, Grid 3 was chosen for further work.

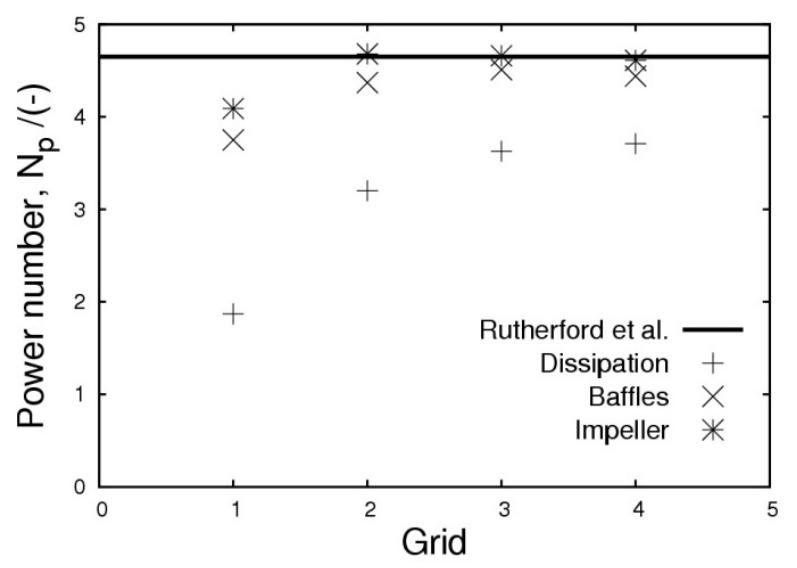

Fig. 2. Power numbers computed via three different approaches; one-phase system

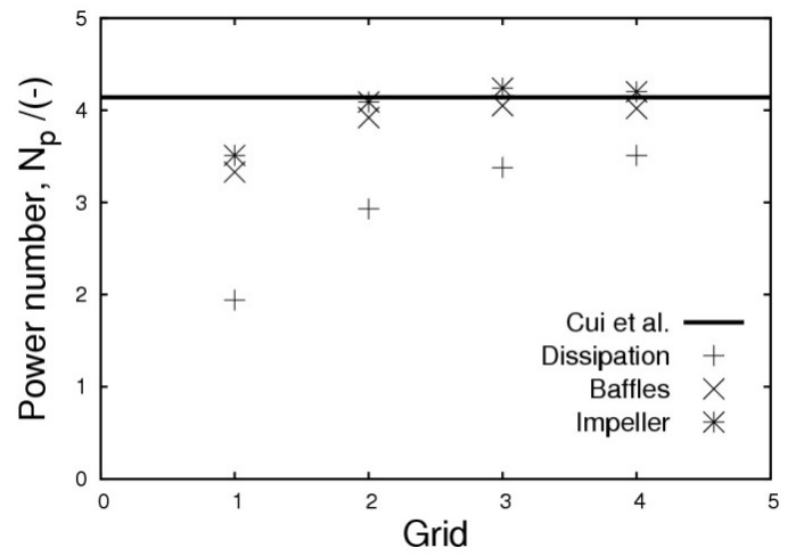

Fig. 3. Power numbers computed via three different approaches; gas flow rate $0.2 \mathrm{vvm}$

\subsection{Gas hold-up}

On the basis of experiments, the global gas hold-up in the system was found to be equal to $(0.70 \pm$ $0.04)$ and $(1.10 \pm 0.05)$ vol.\% for the investigated gas flow rates $(0.2$ and $0.4 \mathrm{vvm}$, respectively), which corresponds well with the correlation for systems with one Rushton turbine given by van't Riet (1975). Qualitative evaluation of the local gas hold-up was performed on the basis of visual observation and is discussed further in the text.

As mentioned earlier, the Schiller-Naumann drag coefficient correlation considers bubbles as perfectly spherical, separate and moving in a stagnant fluid. Because of these assumptions, the predicted gas hold-up was about $40 \%$ lower than the value determined from the experiments. The Ishii-Zuber and Tomiyama models reflect the changes of bubble shape, thus taking into account changes of bubble flow regime. This consideration causes a slight improvement relative to the previous model. However, neglecting the turbulent effects is still the principal issue and results in a $30 \%$ underestimation of the experimental values. All of the previously mentioned models exhibit similar behaviour in stirred tanks: due to underestimated drag, bubbles leave the impeller discharge stream too early, rise through the bulk of the tank upwards without being properly dispersed and exit the system through a small area at the liquid surface (see Fig. 4). 


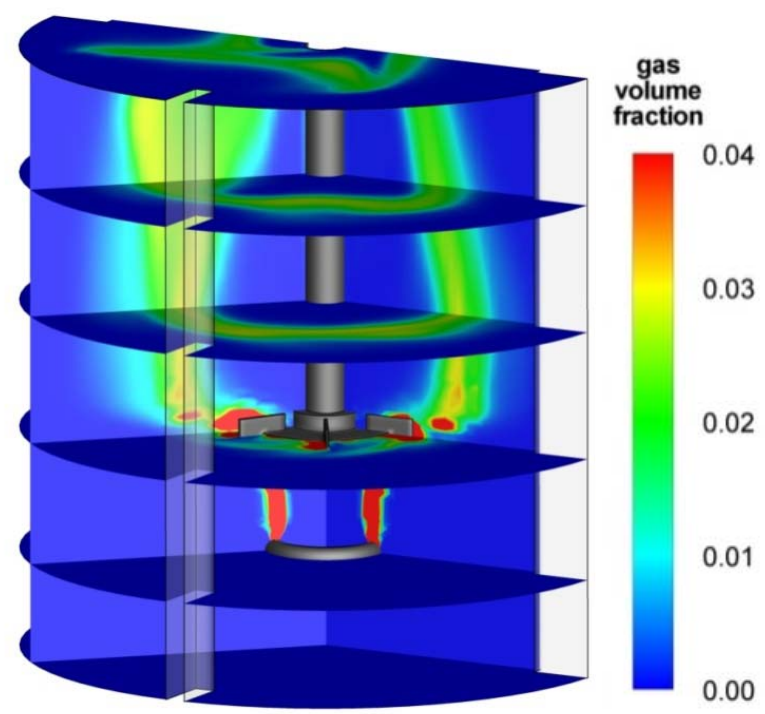

Fig. 4. Contours of gas hold-up using the Ishii-Zuber drag coefficient correlation

To capture the turbulence dampening of slip, a correction needs to be incorporated into the drag coefficient models. The correlation given by Brucato contains an extra term that is added to a drag coefficient in stagnant fluid, $c_{D, 0}$ (Eq.(5)). For computation of $c_{D, 0}$, we used the Tomiyama model (Eq.(4)). The constant $C_{I}$ was adjusted to $2.5 \cdot 10^{-6}$ so that the condition of equality of the simulated and experimental global gas hold-up values was satisfied. However, this model showed serious shortcomings. The extra term appears to overpredict the bubble drag in highly turbulent areas, such as the impeller plane and its close vicinity along the walls. Because of this overprediction, bubbles snatched by the lower recirculation loops are brought under the impeller and trapped there. When they try to slip along the wall, they encounter the highly turbulent area of the impeller plane, gain high drag and are transported back under the impeller. However, bubbles caught by the upper recirculation loops are carried to a significantly less-turbulent area under the liquid surface, where the contribution of the extra term is low and where the bubbles therefore escape the system (Fig. 5). Similar behaviour of this model we can see also in the results published by Ahmed et al. (2010).

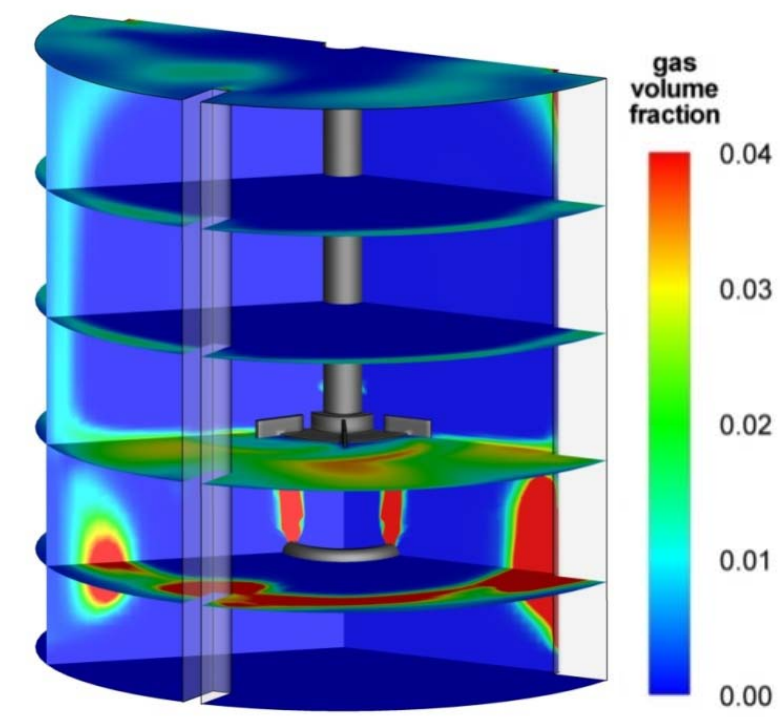

Fig. 5. Contours of gas hold-up using the turbulent drag correction by Brucato

The approach proposed by Bakker is based on modification of the Reynolds number. The constant $C_{2}$ in Eq.(6) was set equal to 0.07. Unfortunately, this value is not universal, and it is dependent on the 
correct prediction of the turbulent field. The simulated gas hold-up distribution is depicted in Fig. 6. Gas is dispersed very well in the upper part of the vessel above the impeller plane, which corresponds to the loading regime. Bubbles from the sparger that enter the impeller region are caught by the impeller discharge stream and are carried away radially towards the wall of the mixing tank. A small fraction of bubbles follows the lower recirculation loops and accumulates behind baffles. The vast majority of bubbles are captured by the upper loops and rise towards the surface along the walls. Some of these bubbles exit the system, and some are transported back to the liquid bulk. However, gas recirculation seems to be overpredicted. This results in increased local gas hold-up in the bulk of the system and subsequently in the SMD overprediction in the region. The obvious reason is that the turbulent correction is dependent on the turbulent viscosity. The value of this variable in a lowturbulent area may be comparable or even bigger than that in a high-turbulent area. The correction may be thus more significant for bubbles located in the bulk of the system than for bubbles flowing in the impeller discharge stream. Such behaviour does not seem to be correct.

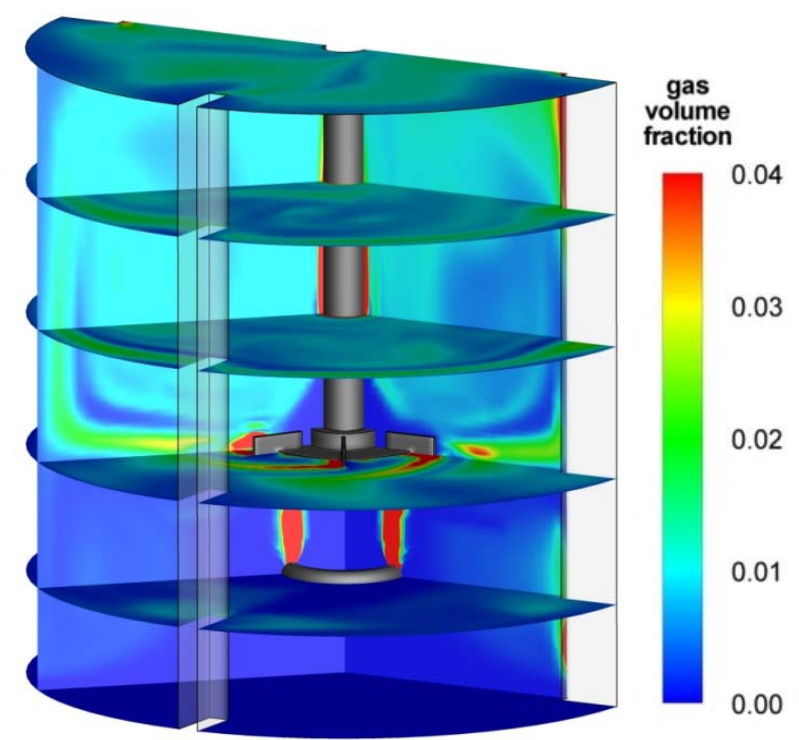

Fig. 6. Contours of gas hold-up using the turbulent drag correction by Bakker

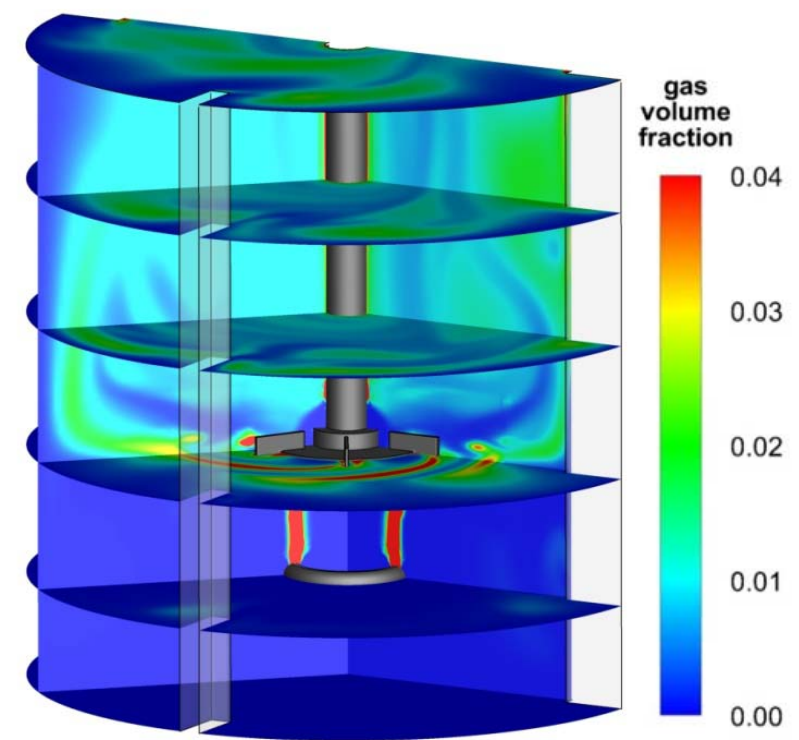

Fig. 7. Contours of gas hold-up using the turbulent drag correction by Montante 
The drag modification introduced by Montante lies in a decrease of the bubble terminal velocity (Eq.(7)). The main drawback of this method proved to be the single value of the bubble terminal velocity in the whole system, irrespective of the local turbulent intensity. No good fit against experimental data was found for a single value. Therefore, a combination of two bubble terminal velocity values was applied $-8 \mathrm{~cm} / \mathrm{s}$ for high turbulent $\left(\varepsilon \geq 1.0 \mathrm{~m}^{2} / \mathrm{s}^{3}\right)$ and $13 \mathrm{~cm} / \mathrm{s}$ for less-turbulent areas $\left(\varepsilon<1.0 \mathrm{~m}^{2} / \mathrm{s}^{3}\right)$ - which produced good agreement with the experimental results (Fig. 7). Nonetheless, a general description of the dependence of the bubble terminal velocity on the local turbulence is needed for more universal application of the model. Petitti et al. (2010) pointed out that the terminal velocity of bubbles is further decreased at high gas hold-ups (above 5\%). This condition is induced by an increased collision frequency between bubbles, which interferes with the rising motion. Because of low gas hold-ups in the studied system, this enhancement was neglected.

\subsection{Bubble size distribution}

During the experiments, 300-500 bubbles were marked and evaluated in each investigated area. The maximum bubble size observed in the system was approximately $6 \mathrm{~mm}$, whereas the smallest detectable bubble diameter was approximately $0.1 \mathrm{~mm}$. Smaller bubbles most likely existed; however, because of limitations imposed by the camera resolution, these bubbles were not incorporated. Such bubbles contain only a very small fraction of the total gas volume and interfacial area. More than $90 \%$ of both the gas volume and the interfacial area were stored in bubbles larger than $1 \mathrm{~mm}$. The average diameter varied from 0.5 to $1.2 \mathrm{~mm}$, and approximately $60 \%$ of the detected bubbles exhibited diameters less than $1 \mathrm{~mm}$.

In the impeller discharge stream, the turbulent dissipation energy has the greatest value, which causes an intensive breakup of bubbles and results in a low value of the SMD (Fig.9). The bubble diameter then slowly increases in the axial direction from the impeller towards the liquid surface and in the radial direction from the vessel wall towards the shaft. Obviously, coalescence prevails in the volume above the impeller plane. The smallest SMD was observed in position A, which was caused by the fact that the system was working in the loading regime when the vast majority of bubbles followed the upper recirculation loops, and only small bubbles were captured by the lower loops. The average diameter of bubbles entering the system was determined to be $8 \mathrm{~mm}$. This value is in good agreement with correlation for inlet bubble size given by Geary and Rice (1991).

Although the drag correction by Montante lacks the dependency on a turbulent quantity, it proved to predict the gas distribution in the system best and it was used for further simulations. As a model describing the disintegration of bubbles, the breakup model by Luo was used and tested. For the first simulations, we discretised the bubble population into 24 intervals with the minimum bubble size, $d_{b, \min }$, equal to $0.1 \mathrm{~mm}$ and left the calibration coefficient of the breakup model, $F_{b r}$, equal to one (Eq.(18)). However, the model severely underestimated the SMD throughout the domain. Practically all of the bubbles were completely smashed into very small particles with diameters close to the size of the smallest group, i.e. $0.1 \mathrm{~mm}$. To understand this behaviour, we have to analyse the DSD function of the breakup model.

Dimensionless DSD curves for three different turbulent dissipation rates are depicted in Fig. 8. We can see that the DSD function is U-shaped and so the model greatly favours the formation of a very small daughter particle and its complement, which, after the breakup process, remains nearly as large as the original mother particle. The equal-sized breakup is considered to be the least probable event. According to the theory by Luo \& Svendsen, breakup occurs whenever the energy of the incoming eddy is higher than the change in the surface energy due to bubble breakup. The smaller the daughter bubble, the smaller the change in the surface energy. It means that when we decrease the minimum bubble size, we decrease the energy that is needed to bring about the breakup into the smallest bubbles 
and so smaller eddies with lower energy are able to pinch off these smallest daughter particles. However, this basic premise of the model is not correct. For breakups with small daughter/mother bubble volume ratios, the capillary pressure is high and even an eddy with a large kinetic energy may not produce enough dynamic pressure to overcome the capillary pressure (Wang et al., 2003).

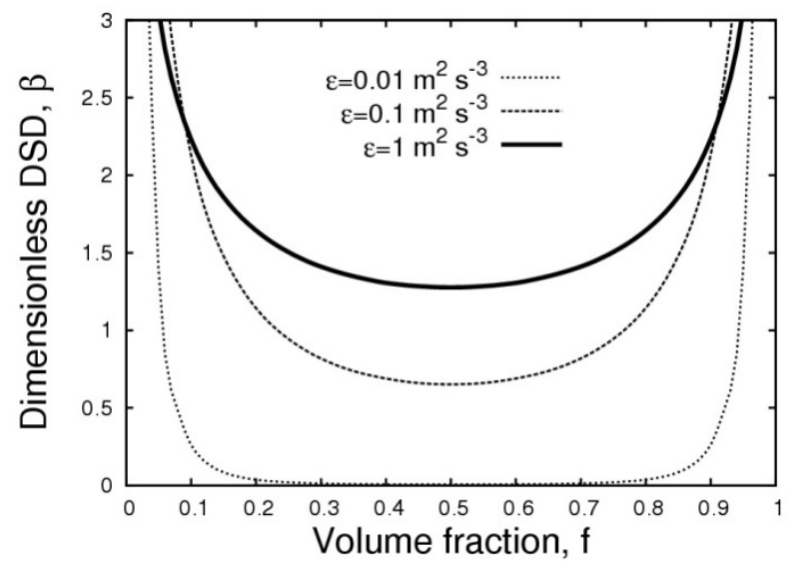

Fig. 8. Evolution of the dimensionless DSD of the Luo \& Svendsen model with the change in the turbulent dissipation energy $\left(d_{i}=0.008 \mathrm{~m}, \alpha=0.01\right)$

Bubbles entering the investigated mixing tank have diameters equal to $8 \mathrm{~mm}$ and are therefore unstable in the turbulent flow of the system and exhibit a high breakup frequency. A large number of small bubbles are gradually peeled off from the breaking mother bubbles while they still maintain high breakup rates. In the end, a large number of bubbles of the smallest size are created, and the resulting SMD is underpredicted.

Obviously, there are two ways how to obtain a better prediction of the bubble size using the presented model - either the breakup rate of bubbles must be decreased by adjustment of the calibration coefficient of the breakup model, or the size of the smallest bubble group must be increased, which results in the formation of larger bubbles by the breakup and so prevents the formation of unreasonably large number of very small bubbles. However, even a ten-fold decrease in the breakup rate resulted in only a slight improvement in the computed SMD, which remained, at all locations, substantially lower than the experimental values (Fig. 9a). A significantly better agreement between simulations and experiments was achieved by setting the size of the smallest bubble group equal to $1.75 \mathrm{~mm}$ (Fig. 9b). The best results were achieved through a combination of both previous attitudes; in our case, the values $F_{b r}=0.12$ and $d_{b, \min }=0.5 \mathrm{~mm}$ were applied. The simulated and experimental values of the bubble SMD were in good agreement at all the investigated points for both gas flow rates (Fig. 9c, 10). Nevertheless, this approach may not seem optimal. Suppressing the formation of particles smaller than the size of the smallest group changes the BSD because small bubbles are not present. However, because a significant majority of the bubble volume and interfacial area (above 95\%) is stored in bubbles larger than $0.5 \mathrm{~mm}$, this method can be used as a good approximation for, e.g., mass transfer simulations, where determination of the interfacial area is the most crucial issue.

This paper should not be taken as an attempt to give any exact coefficients of the studied breakup model but to point out its general features. Although Luo \& Svendsen claimed that their model contains no adjustable parameters, our simulations showed that it is dependent not only on the breakup coefficient of the model, which is a common feature of all breakup models and depends on the successful prediction of the turbulence field, but also on the discretisation of the bubble population, namely on the minimum bubble size, which requires fitting against experimental data and so makes the real applicability of the model questionable. 


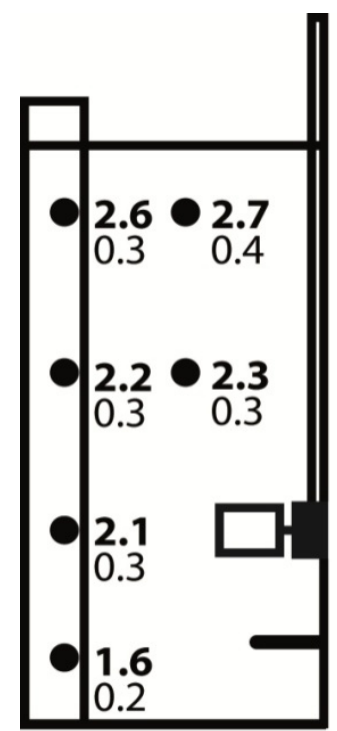

a)

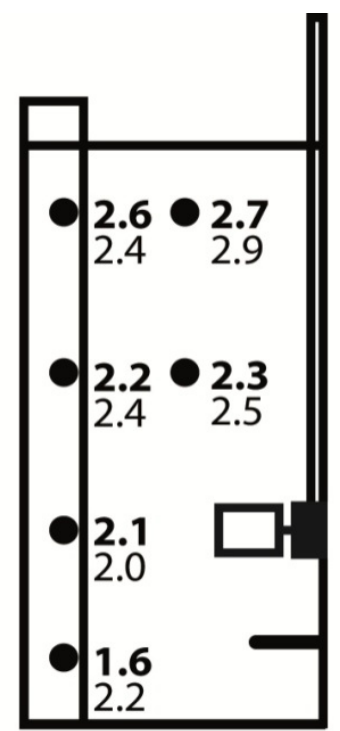

b)

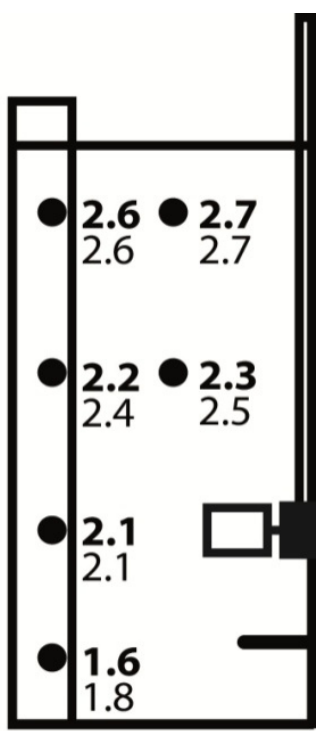

C)

Fig. 9. Comparison of the measured (1st line) and simulated (2nd line) Sauter mean diameter [mm], $Q=0.2 \mathrm{vvm}$

a) The breakup calibration coefficient $F_{b r}=0.1$, the smallest bubble size $d_{b, \min }=0.1 \mathrm{~mm}$

b) The breakup calibration coefficient $F_{b r}=1.0$, the smallest bubble size $d_{b, \min }=1.75 \mathrm{~mm}$

c) The breakup calibration coefficient $F_{b r}=0.12$, the smallest bubble size $d_{b, \min }=0.5 \mathrm{~mm}$

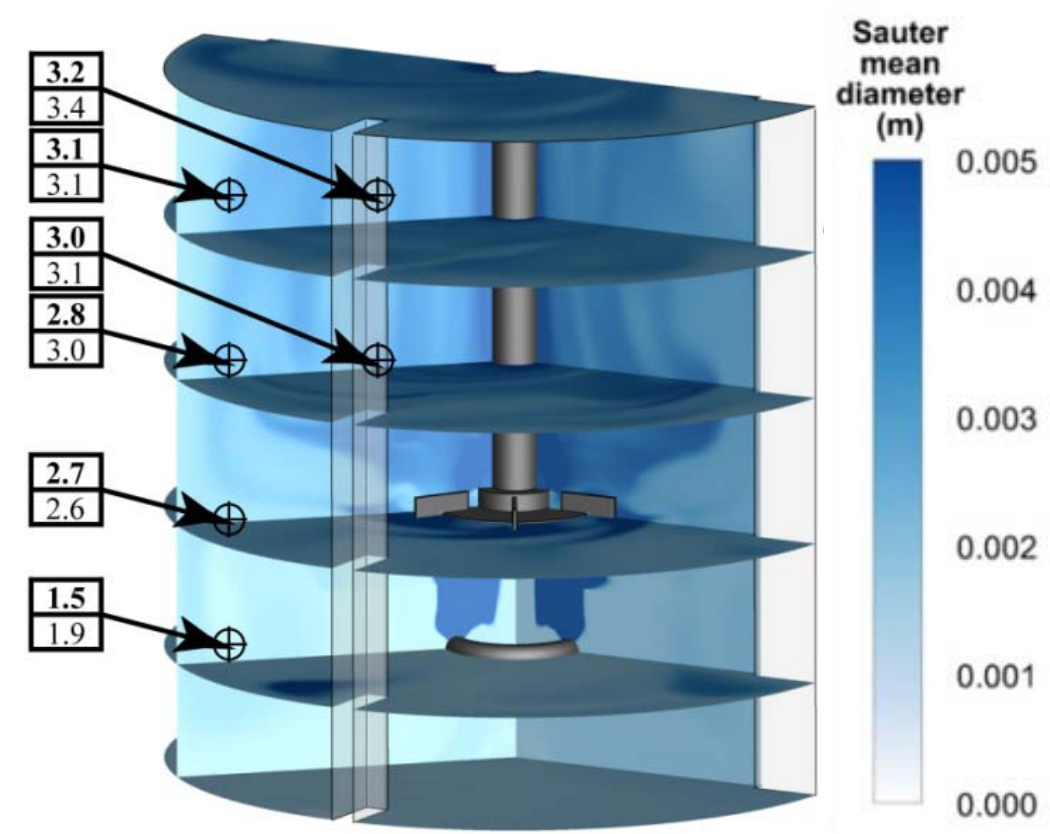

Fig. 10. Contours of the Sauter mean diameter and comparison of the measured $\left(1^{\text {st }}\right.$ line $)$ and modelled $\left(2^{\text {nd }}\right.$ line $)$ values at the investigated points $[\mathrm{mm}], Q=0.4 \mathrm{vvm}, F_{b r}=0.12, d_{b, \min }=0.5 \mathrm{~mm}$

\section{CONCLUSIONS}

In this work, two important characteristics of air-water flow in a stirred tank - gas hold-up and bubble size distribution - were studied both experimentally and numerically. Four computational meshes were tested and their influence on results was investigated. The difference between the real power number and the power number computed from the overall turbulent dissipation rate decreases with the increasing number of computational cells. Obviously, much finer grids than those that have been usually adopted so far for the BSD modelling are required for the correct prediction of turbulent 
quantities in a system. This suggests that the often reported poor predictions of turbulence obtained using the $k-\varepsilon$ turbulence model may be the result of numerical errors rather than deficiencies in the turbulence model. A refinement of the mesh may solve or at least minimise the problem.

Drag coefficient correlations originally derived for non-turbulent systems proved to be unsuitable for prediction of gas hold-up in stirred tanks. Therefore, three turbulent drag corrections were tested for the investigated system. The correlations given both by Brucato and Bakker showed shortcomings in gas distribution predictions. In contrast, the correction by Montante was able to describe global and local gas hold-up in good agreement with experimental data and observations when two values of the bubble terminal velocity were used.

The BSD was measured experimentally using photographic analysis, and these data were used for comparison with numerical results. The breakup model by Luo is able to describe the BSD in stirred tanks correctly but, because of its sensitivity to the size of the smallest bubble group, the model must be adjusted using experimental data and its general applicability is thus limited. Therefore, although the breakup model by Luo is the most widely used model and has been adopted by numerous authors, the use of different breakup models with DSD functions that contain the capillary constraint (Lehr et al., 2002) or prefer the equal-sized breakup (Alopaeus et al., 2002; Martínez-Bazán et al., 1999) should be considered. Besides, modifications of the Luo model that restrict the formation of small bubbles have been proposed (Hagesaether et al., 2002; Wang et al., 2003). These models will be studied together with the local BSD in detail in the upcoming work of the authors.

This project has been supported by the Czech Science Foundation (Grant: 104/09/1290) and by specific university research (Grant: MSMT No 21/2012).

\section{SYMBOLS}

$\begin{array}{ll}a\left(V_{i}, V_{j}\right) & \text { coalescence rate, } \mathrm{m}^{3} \mathrm{~s}^{-1} \\ B_{i} B_{i} & \text { birth rate of bubbles in class } i, \mathrm{~m}^{-3} \mathrm{~s}^{-1} \\ c_{D} & \text { drag coefficient } \\ c_{f} & \text { change in the surface area } \\ C_{\mu} & \text { constant in Eq. } 8 \\ d & \text { diameter, } \mathrm{m} \\ d_{32} & \text { Sauter mean diameter, } \mathrm{m} \\ D_{i} & \text { death rate of bubbles in class } i, \mathrm{~m}^{-3} \mathrm{~s}^{-1} \\ E o E o & \text { Eötvös number } \\ F_{a g}, F_{b r} & \text { calibration coefficients } \\ F_{D, i j} & \text { vector of the drag force, } \mathrm{kg} \mathrm{m}^{-2} \mathrm{~s}^{-2} \\ f & \text { daughter/mother bubble volume ratio } \\ f_{i} & \text { volumetric fraction of class } i \text { in gas phase } \\ g\left(V_{i}\right) & \text { overall breakup rate, } \mathrm{s}^{-1} \\ g\left(V_{i}, V_{j}\right) & \text { partial breakup rate, } \mathrm{s}^{-1} \\ h_{0}, h_{f} & \text { initial, critical thickness of the liquid film separating bubbles, } \mathrm{m} \\ k & \text { turbulent kinetic energy, } \mathrm{m}^{2} \mathrm{~s}^{-2} \\ N_{p} & \text { power number } \\ P_{a g} & \text { collision efficiency } \\ Q & \text { gas flow rate, } \mathrm{m}^{3} \mathrm{~s}^{-1} \\ R e & \text { Reynolds number } \\ T & \text { mixing vessel diameter, } \mathrm{m} \\ & \end{array}$




$$
\begin{array}{ll}
\vec{U} & \text { average velocities vector, } \mathrm{m} \mathrm{s}^{-1} \\
V & \text { volume, } \mathrm{m}^{3}
\end{array}
$$

\section{Greek symbols}

$\begin{array}{ll}\alpha & \text { volume fraction } \\ \beta\left(V_{i} \mid V_{j}\right) & \text { daughter size distribution function, } \mathrm{m}^{-3} \\ \varepsilon & \text { turbulent dissipation energy, } \mathrm{m}^{2} \mathrm{~s}^{-3} \\ \eta & \text { Kolmogorov microscale, } \mathrm{m} \\ \mu, \mu_{t}, \mu_{e f f} & \text { dynamic, turbulent and effective viscosity, Pa s } \\ \xi & \text { dimensionless eddy size } \\ \rho & \text { density, } \mathrm{kg} \mathrm{m}^{-3} \\ \sigma & \text { surface tension, } \mathrm{N} \mathrm{m} \mathrm{m}^{-1} \\ \omega_{a g} & \text { collision frequency, } \mathrm{m}^{3} \mathrm{~s}^{-1}\end{array}$

\section{Subscripts}

$a g, b r \quad$ aggregation, breakup

$b$

bubble

$g, l \quad$ gas, liquid phase

$i, j \quad$ index of a bubble size group

\section{REFERENCES}

Ahmed S.U., Ranganathan P., Pandey A., Sivaraman S., 2010. Computational fluid dynamics modeling of gas dispersion in multi impeller bioreactor. J. Biosci. Bioeng., 109, $\overline{588} \overline{-}-597$. DOI: $10 . \overline{101} / \mathrm{j}$.jbiosc.2009.11.014.

Alopaeus V., Koskinen J., Keskinen K. I., 1999. Simulation of the population balances for liquid-liquid systems in a nonideal stirred tank. Part 1 - Description and qualitative validation of the model. Chem. Eng. Sci., 54, 5887-5899. DOI: 10.1016/S0009-2509(99)00170-0.

Alopaeus V., Koskinen J., Keskinen K. I., Majander J., 2002. Simulation of the population balances for liquidliquid systems in a nonideal stirred tank. Part 2 - parameter fitting and the use of the multiblock model for dense dispersions. Chem. Eng. Sci., 57, 1815-1825. DOI: 10.1016/S0009-2509(02)00067-2.

ANSYS, Inc., 2012. CFX-Solver Theory Guide, Release 14.5. Canonsburg, PA, USA.

Aubin J., Fletcher D. F., Xuereb C., 2004. Modeling turbulent flow in stirred tanks with CFD: the influence of the modeling approach, turbulence model and numerical scheme. Exp. Therm. Fluid Sci., 28, 431-445. DOI: 10.1016/j.expthermflusci.2003.04.001.

Bakker A., van den Akker H.E.A., 1994. A computational model for the gas-liquid flow in stirred reactors. Chem. Eng. Res. Des., 72, 594-606.

Brucato A., Grisafi F., Montante G., 1998. Particle drag coefficients in turbulent fluids. Chem. Eng. Sci., 53, 3295-3314. DOI: 10.1016/S0009-2509(98) $\overline{001} 14-6$.

Coroneo M., Montante G., Paglianti A., Magelli F., 2011. CFD prediction of fluid flow and mixing in stirred tanks: Numerical issues about the RANS simulations. Comput. Chem. Eng., 35, 1959-1968. DOI: 10.1016/j.compchemeng.2010.12.007.

Cui Y.Q., van der Lans R.G.J.M., Luyben K.C.A.M., 1996. Local power uptake in gas-liquid systems with single and multiple Rushton turbines. Chem. Eng. Sci., 51, 2631-2636. DOI: 10.1016/0009-2509(96)00128-5.

Deglon D.A., Meyer C. J., 2006. CFD modelling of stirred tanks: Numerical considerations. Miner. Eng., 19, 1059-1068. DOI: $10.1016 /$ j.mineng.2006.04.001.

Frank T., Zwart P.J., Krepper E., Prasser H.-M., Lucas D., 2008. Validation of CFD models for mono- and polydisperse air-water two-phase flows in pipes. Nucl. Eng. Des., 238, 647-659. DOI: 10.1016/j.nucengdes.2007.02.056.

Geary N. W., Rice R. G., 1991. Bubble size prediction for rigid and flexible spargers. DOI: $10.1002 /$ aic.690370202.

Hagesaether L., Jakobsen H. A., Svendsen H. F., 2002. A model for turbulent binary breakup of dispersed fluid particles. Chem. Eng. Sci., 57, 3251-3267. DOI: 10.1016/S0009-2509(02)00197-5. 
Ishii M., Zuber N., 1979. Drag coefficient and relative velocity in bubbly, droplet or particulate flows. AIChE J., 25, 843-855. DOI: 10.1002/aic.690250513.

Kerdouss F., Bannari A., Proulx P., Bannari R., Skrga M., Labrecque Y., 2008. Two-phase mass transfer coefficient prediction in stirred vessel with a CFD model. Comput. Chem. Eng., 32, 1943-1955. DOI: 10.1016/j.compchemeng.2007.10.010.

Khopkar A. R., Rammohan A. R., Ranade V.V., Dudukovic M. P., 2005. Gas-liquid flow generated by a Rushton turbine in stirred vessel: CARPT/CT measurements and CFD simulations. Chem. Eng. Sci., 60, 2215-2229. DOI: 10.1016/j.ces.2004.11.044.

Kumar S., Ramkrishna D., 1996. On the solution of population balance equations by discretization - I. A fixed pivot technique. Chem. Eng. Sci., 51, 1311-1332. DOI: 10.1016/0009-2509(96)88489-2.

Laakkonen M., Moilanen P., Alopaeus V., Aittamaa J., 2007. Modelling local bubble size distributions in agitated vessels. Chem. Eng. Sci., 62, 721-740. DOI: 10.1016/j.ces.2006.10.006.

Lehr F., Millies M., Mewes D., 2002. Bubble-size distributions and flow fields in bubble columns. $A I C h E J ., 48$, 2426-2443. DOI: 10.1002/aic.690481103.

Liao Y., Lucas D., 2010. A literature review on mechanisms and models for the coalescence process of fluid particles. Chem. Eng. Sci., 65, 2851-2864. DOI: 10.1016/j.ces.2010.02.020.

Lo S., 1996. Application of the MUSIG model to bubbly flows. AEAT-1096, AEA Technology.

Luo H., Svendsen H.F., 1996. Theoretical model for drop and bubble breakup in turbulent dispersions. 42, 1225-1233. DOI: 10.1002/aic.690420505.

Marchisio D.L., Fox R.O., 2013. Computational models for polydisperse particulate and multiphase systems. Cambridge University Press, New York, USA.

Martínez-Bazán C., Montanes J.L., Lasheras J.C., 1999. On the breakup of an air bubble injected into fully developed turbulent flow. Part 1. Breakup frequency. J. Fluid Mech., 401, 157-182. DOI: 10.1017/S0022112099006680.

Montante G., Horn D., Paglianti A., 2008. Gas-liquid flow and bubble size distribution in stirred tanks. Chem. Eng. Sci., 63, 2107-2118. DOI: 10.1016/j.ces.2008.01.005.

Montante G., Paglianti A., Magelli F., 2007. Experimental analysis and computational modelling of gas-liquid. stirred vessels. Chem. Eng. Res. Des., 85, 647-653. DOI: $10.1205 / \mathrm{cherd} 061 \overline{41}$.

Petitti M., Nasuti A., Marchisio D.L., Vanni M., Baldi G., Mancini N., Podenzani F., 2010. Bubble size distribution modeling in stirred gas-liquid reactors with QMOM augmented by a new correction algorithm. AIChE J., 56, 36-53. DOI: 10.1002 /aic.12003.

Prince M.J., Blanch H.W., 1990. Bubble coalescence and break-up in air-sparged bubble columns. 1485-1499. DOI: 10.1002/aic.690361004.

Rakoczy R., Masiuk S., 2009. Experimental study of bubble size distribution in a liquid column exposed to a rotating magnetic field. Chem. Eng. Process., 48, 1229-1240. DOI: 10.1016/j.cep.2009.05.001.

Ranganathan P., Sivaraman S., 2011. Investigations on hydrodynamics and mass transfer in gas-liquid stirred reactor using computational fluid dynamics. Chem. Eng. Sci., 66, 3108-3124. DOI: 10.1016/j.ces.2011.03.007.

Rutherford K., Mahmoudi S.M.S., Lee K.C., Yianneskis M., 1996. The influence of Rushton impeller blade and disk thickness on the mixing characteristics of stirred vessels. Chem. Eng. Res. Des., 74, 369-378.

Sato Y., Sekoguchi K., 1975. Liquid velocity distribution in two-phase bubble flow. Int. J. Multiphase Flow, 2, 79-95. DOI: 10.1016/0301-9322(75)90030-0.

Scargiali F., D'Orazio A., Grisafi F., Brucato A., 2007. Modelling and simulation of gas-liquid hydrodynamics in .

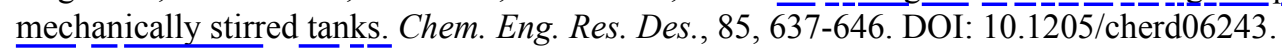

Schiller L., Naumann Z., 1935. A drag coefficient correlation. Z. Ver. Deutscher Ing., 77, 318.

Selma B., Bannari R., Proulx P., 2010. Simulation of bubbly flows: Comparison between direct quadrature method of moments (DQMOM) and method of classes (CM). Chem. Eng. Sci., 65, 1925-1941. DOI: 10.1016/j.ces.2009.11.018.

Tomiyama A., Kataoka I., Zun I., Sakaguchi T., 1998. Drag coefficients of single bubbles under normal and microgravity conditions. JSME Int. J. Series B, 41, 472-479. DOI: 10.1299/jsmeb.41.472.

van't Riet K., 1975. Turbine agitator hydrodynamics and dispersion performance. Ph.D. thesis, University of Delft, The Netherlands.

Wang T., Wang J., Jin Y., 2003. A novel theoretical breakup kernel function for bubbles/droplets in a turbulent flow. Chem. Eng. Sci., 58, 4629-4637. DOI: 10.1016/j.ces.2003.07.009. 
CFD prediction of gas-liquid flow in an aerated stirred vessel using the population balance model

Received 29 October 2013

Received in revised form 15 January 2014 Accepted 16 January 2014 